Brit.J. Ophthal. (1952) 36, . 518

\title{
CYCLODIALYSIS COMBINED WITH POSTERIOR TREPHINING * +
}

BY

\author{
H. J. FLIERINGA \\ Eye Hospital, Rotterdam
}

IN the treatment of certain cases of glaucoma, most Dutch ophthalmologists are inclined to perform cyclodialysis, either in its original form as described by Heine or by the Blascovics method (cyclodialysis inversa). I now propose to discuss not the results of this operation, but its technique, which has never given me entire satisfaction. It is especially the necessary scleral incision, simple as it may appear, which I do not consider very easy to perform. If extreme caution is observed, the sclera may not be incised deep enough and it may therefore prove difficult to introduce a cyclodialysis spatula. In other cases, not only the sclera but also the choroid and retina may become perforated with consequent loss of vitreous. If, in such cases, a cyclodialysis spatula is introduced, it will not always move in the suprachoroidal space towards the limbus, but, before appearing in the angle of the anterior chamber, will penetrate through the ciliary body instead of tearing loose the sclera. I have therefore attempted to apply a different technique by substituting scleral trephining for scleral incision. This trephining is performed by means of a $1.5-\mathrm{mm}$. trephine under the insertion of one of the recti muscles (preferably the superior rectus, if it is desired to perform cyclodialysis in the upper area). The anterior part of the rectus muscle is exposed, a longitudinal incision is made, and both halves of the muscle are separated by sutures (see Figure). Thus a distinct triangle of the sclera becomes visible where trephining is performed.

Taking into consideration that the sclera is usually much thinner under the muscular insertion than elsewhere, I prefer for this purpose not Elliot's trephine but one which has a guard attached to it. The guard is set at a depth of $0.5 \mathrm{~mm}$. which is in most cases quite sufficient. A scleral disk can thus be punched out whole without the use of scissors and without damage to the choroid. From the trephine opening onwards the ciliary body is torn loose in the usual manner. One should take care, however, to use a spatula which is somewhat longer than usual, the distance from the trephine hole to the limbus being about two millimetres greater. Apart from the technique, this procedure presents the following advantages:

The supra-choroidal and subconjunctival spaces are at once brought into communication with each other, and liquid can thus filter from the eyeball.

* Paper read to the Netherlands Ophthalmologichl Society, September, 1951.

+ Received for publication June. 5, 1952. 
The insertion of the rectus muscle, which should be carefully replaced, prevents a prolapse of the choroid through the trephine opening. Furthermore, I assume (without being able to offer absolute proof-no eye thus operated on having as yet been dissected) that, owing to the movements of the rectus muscle, the trephine opening will less easily become obliterated, filtering from the eyeball being thus facilitated.

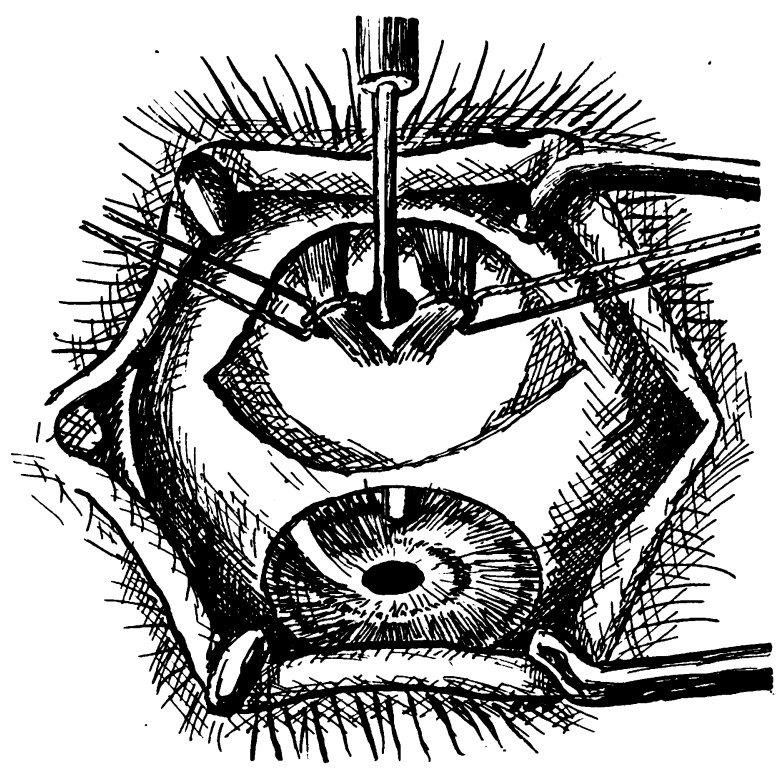

FIGURE.-Exposure of sclera by separation of rectus muscle, and insertion of trephine.

AdDENDUM.-After delivering the above paper, I came across an article by Sallmann (1935) entitled "Die Trepanationszyklodialyse, eine Abänderung der Heineschen Zyklodialysenoperation ". It appears that Sallmann has also adopted a modified technique, for both practical and functional reasons. By Sallmann's method, however, the filtering hole between the supra-choroidal and subconjunctival spaces becomes obliterated quite quickly.

It remains to be seen what will be the final fate of a trephine hole situated beneath the rectus muscle. This can only be judged after my method has been used in a sufficiently large number of cases and has been tested both clinically and pathologically. As far as I know, Sallmann's method has hardly ever been applied in the Netherlands.

\section{REFERENCE}

Sallmann, L. (1935). Z. Augenheilk., 86, 111. 